\section{Seit 1. Juli herrscht Reform im Labor}

D ie neue Laborreform betrifft auch allergologisch tätige Ärzte und insbesondere die mit eigenem Labor. Die Berechnung von Labor-Grundpauschalen und Wirtschaftlichkeitsbonus ändert sich Fachgruppen-spezifisch nicht. Um die Fachgruppen-spezifischen Punktzahlen des Wirtschaftlichkeitsbonus unverändert belassen zu können, wurden die Umrechnungsfaktoren von DM-Kostensätzen auf Punktzahl-Volumina angepasst. So ändert sich der Umrechnungsfaktor

— für den OI/II-Bereich von 13,5 auf 13,9

— für den O/III-Bereich von 16,1 auf 14,6 .

Aufgrund der Umstellung in EURO zum 1. Januar 2002 werden sich die Umrechnungsfaktoren für OI/II auf 26,6 und O/III auf 28,6 ändern. Der $24 \%$ ige Honoraraufschlag für den O/III-Bereich gilt nicht mehr.

Auch für allergologisch tätige Ärzte kommt es im O/III-Bereich zu einer teilweisen Neubewertung der DM-Kostensätze, wobei die Kosten für die Ziffer 4310 (= quantitative Bestimmung von Gesamt-IgE) von 12 auf 9 DM gesunken ist. Die Kosten für folgende Ziffern sind gleich geblieben:

$-4198=$ Theophyllin-Bestimmung

- 4314 = Untersuchung auf Allergenspezifische Immunglobuline in Einzelansätzen bis zu zehn Allergenen oder Allergenmischung je Ansatz

— 4315 = Untersuchung auf Allergenspezifische Immunglobuline in Einzelansätzen, jede weitere, über die Leistung nach Nr. 4314 hinausgehende Bestimmung bis insgesamt 15 Allergenen (bzw. Mischung) je Ansatz

$-4320=$ Untersuchung auf Allergenspezifische Immunglobuline mindestens 20 deklarierten Allergenen auf einem Träger und Differenzierung nach Einzel-Allergenen

-4325 = qualitativer Nachweis von humanem Protein mittels Immunpräzipitation je Nachweis unter Angabe des Proteins

Die Kosten für die Ziffer 4244 (= eosinophiles kationisches Protein) sind von 35 auf 43,50 DM angehoben worden.
Für allergologisch tätige Ärzte gibt es eine wichtige Ausnahme-Kennziffer: die Nr. 3484 = allergische Erkrankung bei Kindern bis zum vollendeten sechsten Lebensjahr. Diese Ziffer sollte im Sinne einer kompletten Budget-Befreiung des Einzelfalles zwingend angesetzt werden. Hier fallen auch alle anderen Laborleistungen innerhalb desselben Behandlungsfalls nicht unter die Budgetbelastung - ob sie nun ursächlich mit der Ausnahme-Kennziffer in $\mathrm{Zu}$ - sammenhang stehen oder nicht. Wenn die Ziffer 3484 wegen Allergie-Diagnostik bei einem bis zu sechs Jahre alten Kind zur Abrechnung kommt, bedeutet dies, dass auch alle anderen Blutuntersuchungen wie z.B. Blutbild, Eisen usw. nicht $\mathrm{zu}$ einer Budget-Belastung führen.

Unterm Strich wird es somit für die allergologisch tätigen Ärzte EinnahmeMinderungen geben, die insbesondere durch die Abwertung des Gesamt-IgE's und den Wegfall des 24\%ige Honoraraufschlags für den O/III Bereich bedingt sind. Dr.W. Kersten, Moers

\title{
Überschreiten von Arzneimittelgrößen
}

Bi ei den Erkrankungen Asthma bronchiale und COPD müssen wir in der Regel Dosier-Aerosole oder Pulver-Inhalatoren verordnen. Meist handelt es sich um eine lebenslange Therapie, die in der Regel hohe Kosten verursacht. Nicht für alle Patienten ist die gleiche Applikationsform möglich. Die Deposition der unterschiedlichen Präparate ist zu berücksichtigen, die bei den neuen Devices bis zu 20fach höher liegen im Vergleich zu den scheinbar billigeren Dosier-Aerosolen. Die Prüfer in den KV'en müssten die Kosten und die Wirksamkeit der einzelnen Präparate berücksichtigen. Im Endeffekt sind die neuen Produkte mit zweimaliger Anwendung pro Tag billiger als eine $\mathrm{Be}$ darfs-Inhalation von teils 10 bis $20 \mathrm{Hü-}$ ben eines Dosier-Aerosols. Durch eine zeitgemäße Behandlung reduzieren sich automatisch die Kosten für Krankenhausaufenthalte, Arbeitsunfähigkeiten, Reha-Maßnahmen usw.

Die Begutachter dürfen die Medikamentenkosten der einzelnen Krankheitsbilder nicht einseitig betrachten, vielmehr müssen sie die Gesamtschau als Begründung für den Widerspruch anführen. Ganz wichtig ist die Erwähnung von Praxis-Besonderheiten, und hier speziell der spezifischen Immuntherapie (SIT). Jeder Kollege sollte sich im Fall einer Prüfung bei Überschreitung der Arzneimittelgrößen von seiner KV die Quartalsbilanz und die Arznei-Patienten-Tabellen zuschicken lassen. Von dem Verfahren ausgenommen sind Patienten, die der Polizei, Bundeswehr, Kriegsopferversorgung, Bundespost oder dem Grenzschutz angehören sowie Asylbewerber und Sozialhilfe-Empfänger. Die Kosten der Hyposensibilisierungs-Lösungen sind zu addieren und müssen von dem Gesamt-Verordnungsvolumen abgezogen werden. Dann sieht die Sache in der Regel schon ganz anders aus. Bei der Begründung sollte auf die Wirtschaftlichkeit der SIT hingewiesen werden. Eine mögliche Hilfestellung hierfür sind die Arbeiten von Büchner und Siepe [Allergo J 1995; 4: 156-63] bzw. von Märtens und Lobermeyer in dieser Ausgabe.

Die Frage stellt sich, warum man sich auf einmal mit dem neuen Quälinstrument Arzneimittel-Richtgrößen für die bewährten Therapieformen rechtfertigen muss. Gebeutelt sind insbesondere die Kollegen, die viele Patienten behandeln und dies auch angemessen tun. Die Folge solcher Maßnahmen ist schon heute eine 3-Klassen-Medizin.

Man darf sich auf der Regierungsseite - egal welcher Couleur - nicht wundern, dass ein System, das über Jahre gezielt heruntergefahren wird, nicht durch „Reformen“" innerhalb von Monaten gesundet. Das heißt wiederum: Wir haben uns für die nächsten Jahre auf die Mangelverwaltung einzustellen. Wunder sind bei den chronisch knappen Kassen nicht zu erwarten!

Dr.W. Kersten, Moers 